\title{
Ressecção de um Gliossarcoma com Cirurgia Acordada para Mapeamento da Linguagem
}

\section{Resection of a Gliosarcoma Using Awake Language Mapping}

Catarina Pessanha Viegas ${ }^{1}$, João Leote², Ana Silvestre², António Pérez-Hick³, Luísa Albuquerque ${ }^{2}$, Manuel Cunha e Sá1

\section{RESUMO}

A ressecção cirúrgica tem um papel fulcral no tratamento dos gliomas de alto grau, no qual se incluem os gliossarcomas. A cirurgia deve ser realizada conhecendo a neuroanatomia funcional e a proximidade do tumor com as áreas eloquentes, para diminuir a morbilidade cirúrgica.

Reportamos o caso de uma doente de 66 anos com alterações da linguagem devido à presença de uma lesão intra-axial fronto-opercular e insular esquerda. Foi realizada a ressecção cirúrgica desta lesão com mapeamento cerebral da linguagem e da função motora, com a doente acordada. A remoção foi total, sem indução de défices neurológicos definitivos de novo.

A cirurgia com o doente acordado com eletroestimulação cortico-subcortical constitui uma abordagem segura na ressecção máxima de gliomas de alto grau, preservando a função e otimizando a qualidade de vida.

PALAVRAS-CHAVE: Gliossarcoma; Mapeamento Cerebral; Monitorização Neurofisiológica Intraoperatória; Neoplasias do Cérebro/cirurgia; Procedimentos Neurocirúrgicos; Vigília

\section{ABSTRACT}

Surgical resection plays a central role in the management of high grade gliomas, including gliosarcomas. Surgery must be performed knowing the functional neuroanatomy and its relation to the tumor, in order to diminish the surgical morbidity.

We report the case of a 66-year-old patient with language disturbances due to an intra-axial mass lesion located in the left frontal-opercular and insular region. The resection was performed using awake language and motor mapping. It was a total resection with no post-surgical new permanent neurological deficit.

Intraoperative cortical-subcortical stimulation in the awake patient represents a safer approach to high grade glioma resection preserving function, and thus, optimizing quality of life.

KEYWORDS: Brain Mapping; Brain Neoplasms/surgery; Gliosarcoma; Intraoperative Neurophysiological Monitoring; Neurosurgical Procedures; Wakefulness

1. Neurocirurgia, Hospital CUF Infante Santo, Lisboa, Portugal. 2. Serviço de Neurologia, Hospital CUF Infante Santo, Lisboa, Portugal. 3. Serviço de Anestesiologia, Hospital CUF Infante Santo, Lisboa, Portugal.

Recebido: 03/05/2017 - Aceite: 01/08/2017 


\section{INTRODUÇÃO}

Os gliossarcomas são tumores cerebrais malignos, correspondendo a cerca de $4 \%$ dos gliomas de alto grau. São compostos por elementos de diferenciação gliomatosa e mesenquimatosa. ${ }^{1-4} \mathrm{Na}$ prática clínica os gliossarcomas são tratados como os glioblastomas, com uma combinação de cirurgia seguida de radioterapia e quimioterapia. A sobrevida média situa-se entre os 9 e os 16 meses. Estudos recentes evidenciam que a extensão da ressecção cirúrgica está diretamente relacionada com a sobrevida livre de doença e sobrevida global nos gliomas de alto grau. ${ }^{2,3}$

Conhecendo este benefício da extensão da ressecção, é imperativo que a intervenção cirúrgica seja realizada de uma forma segura com o objetivo de preservar a qualidade de vida. É ponderada a realização de uma cirurgia com o doente acordado se o tumor se encontra na proximidade de áreas eloquentes, nomeadamente com função de linguagem. ${ }^{5}$ A seleção criteriosa do doente deve ser realizada tendo em conta as características imagiológicas do tumor, as comorbilidades do doente e o estado neurológico pré-operatório.

É reportada a ressecção cirúrgica de um gliossarcoma fronto-opercular esquerdo, utilizando eletroestimulação cortico-subcortical, com o doente acordado.

\section{CASO CLÍNICO}

Uma mulher de 66 anos, sem antecedentes relevantes, teve um episódio de afasia global autolimitado, correspondendo a uma crise convulsiva parcial simples, sem recuperação total, mantendo um défice de linguagem, com hesitação no início do discurso e pausas anómicas.

Realizou ressonância magnética (RM) cranioencefálica que documentou uma volumosa lesão expansiva intra-axial fronto-opercular e insular esquerda com intenso reforço de sinal após gadolínio; e três outras lesões intra-axiais de pequenas dimensões, duas anteriores frontais e outra interna em relação à lesão principal (Fig. 1).

A RM funcional demonstrou ativação cortical para as tarefas de linguagem na região fronto-opercular esquerda, em estreita proximidade com a lesão de maiores dimensões (Fig. 2).

Na avaliação neuropsicológica apresentava ligeiras alterações da nomeação, iniciativa verbal oral semântica e fonológica e atenção.

Foi proposta a intervenção cirúrgica, que devido aos sintomas experienciados pela doente e à localização da lesão na região fronto-opercular esquerda, foi decidido realizá-la com a doente acordada, com mapeamento funcional intraoperatório.

A cirurgia foi realizada com a técnica asleep/awake/asleep,
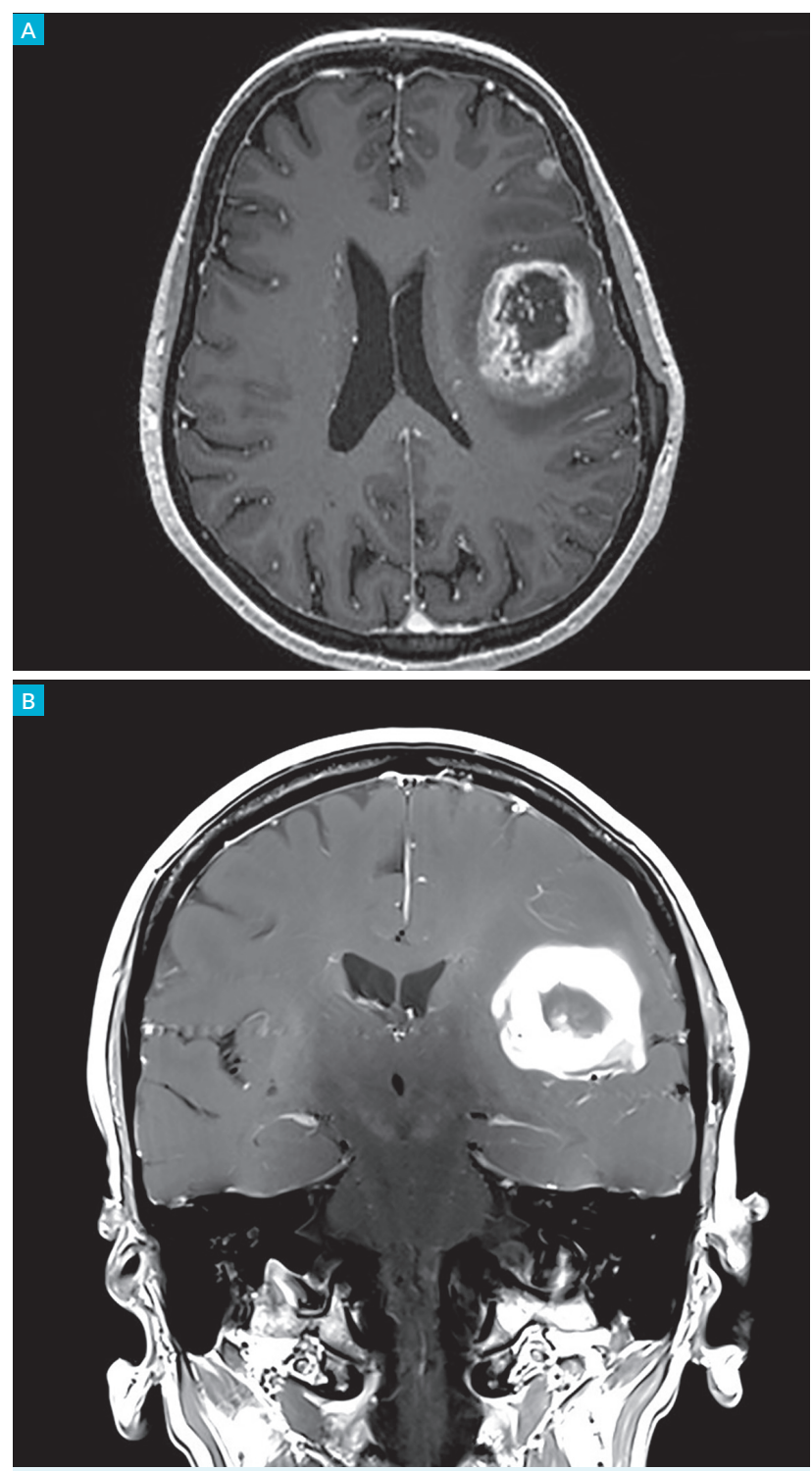

FIGURA 1. RM cranioencefálica pré-operatória (sequência T1 com gadolínio), documentando uma lesão intra-axial fronto-opercular e insular esquerda em axial (A) e coronal (B).

com identificação da localização das lesões com neuronavegação. Foi utilizada uma estimulação de baixa frequência com um elétrodo bipolar com espaçamento de 5 mm entre as pontas (frequência de pulso $50 \mathrm{~Hz}$; duração de fase de pulso singular de $1 \mathrm{~ms}$; amplitude $1-6 \mathrm{~mA})^{5,6} \mathrm{e}$ um elétrodo monopolar de elevada frequência com uma sequência de 5 pulsos $(250 \mathrm{~Hz}, 500 \mathrm{~ms}),{ }^{7}$ que foram aplicados a nível cortico-subcortical. Foi inicialmente realizada uma prova de contagem 1-10 para mapeamento sensitivo-motor ( $n^{\circ} 1,2$, 3; Fig. 3), tendo-se verificado paragem do discurso com 2,5 mA. Em seguida a doente foi testada com a prova de nomeação, provocando anomia com a estimulação ao nível da pars triangularis, área de Broca ( $n^{\circ} 4$; Fig. 3).

Removeu-se uma pequena lesão frontal anterior e a lesão de maiores dimensões através da dissecção da porção posterior da vala sílvica (Fig. 3), tendo em conta os limites funcionais, quer a nível cortical, quer a nível sub- 


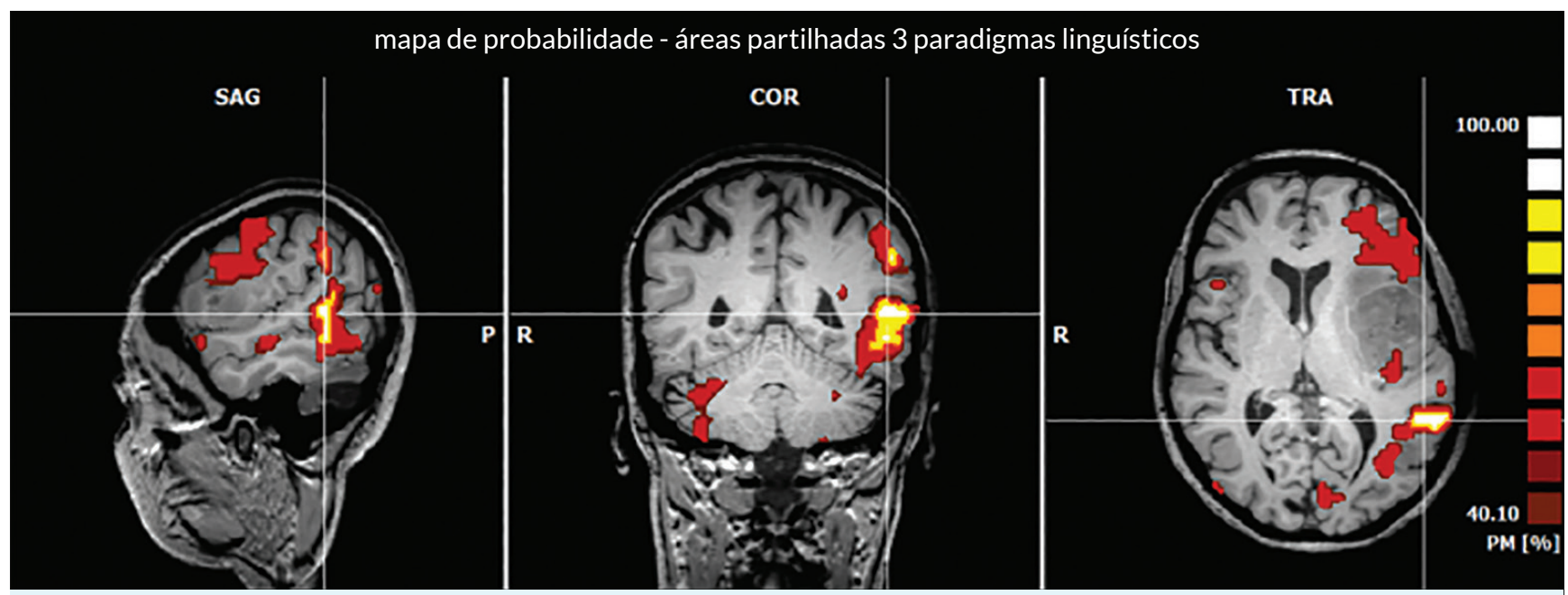

FIGURA 2. RM funcional, mostrando a proximidade das áreas da linguagem a lesão intra-axial.

cortical, mantendo a estimulação durante a ressecção tumoral, testando continuamente a linguagem e a função motora, com identificação da via piramidal a cerca de 3 mm da margem póstero-interna da ressecção.

No pós-operatório, a doente sofreu um agravamento dos défices neurológicos até às 48 horas, com alterações da nomeação, da compreensão verbal, escrita e leitura. Houve uma recuperação até ao estado neurológico pré-operatório, após a reabilitação com terapia da fala, no período de um mês.

A RM cranioencefálica pós-operatória (24 horas) documentou uma remoção total da lesão de maiores dimensões e de uma das lesões frontais anteriores (Fig. 4).

O diagnóstico histológico foi de gliossarcoma grau IV.

\section{DISCUSSÃO}

Os gliossarcomas são tumores raros, com um prognóstico pior do que os glioblastomas. Existem poucas publicações relativamente a este tipo de gliomas. ${ }^{1,4}$ A sobrevida média situa-se entre os 6 e os 16 meses. A abordagem terapêutica é semelhante à dos glioblastomas, combinando a ressecção cirúrgica com a radioterapia e quimioterapia (STUPP ${ }^{8}$ ).

Apesar de extremamente agressivos, os gliomas de alto grau têm uma maior sobrevida global quanto maior for a remoção do tumor. 3,9,10 A remoção total deve ser equacionada tendo em conta a preservação da função motora, linguagem e funções cognitivas. $\bigcirc$ mapeamento cerebral intraoperatório com cirurgia acordada constitui a técnica gold standard para ressecção tumoral com manutenção da função em áreas eloquentes cortico-subcorticais, diminuindo a morbilidade cirúrgica. 2,3,5,6,11 A escolha do doente deve ser criteriosa de modo a assegurar um bom resultado funcional, sendo que doentes com graves alterações da linguagem pré-operatória não são candidatos (mais de 25\% de erros na prova de nomeação).
No caso acima descrito a doente apresentou-se com alterações da linguagem que melhoraram com a corticoterapia pré-operatória, o que permitiu uma colaboração eficaz na avaliação pré-operatória, levando à escolha da realização da cirurgia com a doente acordada. Foi possível uma remoção total da lesão de maiores dimensões tendo em conta os limites funcionais, após o mapeamento cerebral. Houve um agravamento da linguagem nas primeiras 48 horas após a intervenção cirúrgica, com recuperação para o estado pré-operatório após um mês de reabilitação funcional.

O agravamento do défice de linguagem no pós-operatório está descrito em 14\% - 50\% dos doentes com uma indução de défices definitivos em apenas 0\% - 2,4\% dos casos. $^{2,3}$

Outras funções cognitivas superiores podem ser testadas durante a cirurgia acordada de acordo com a avalia-

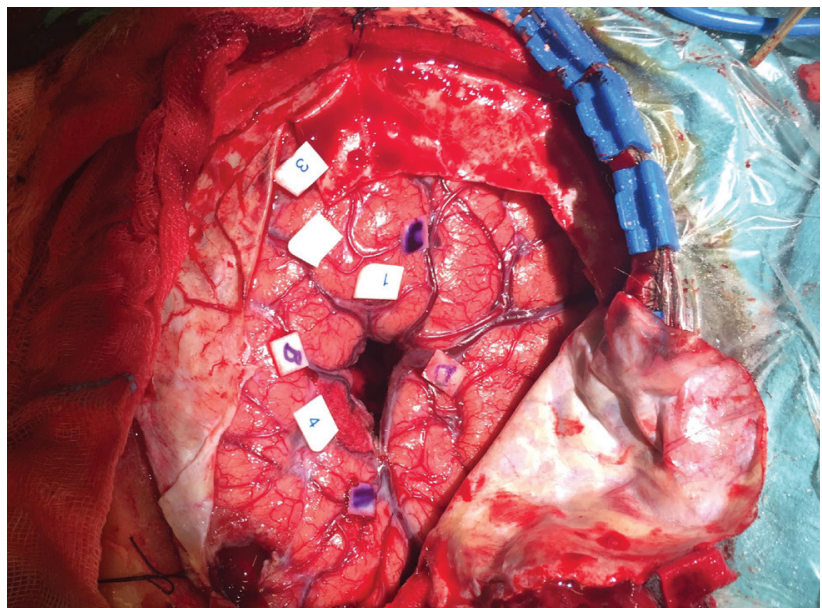

FIGURA 3. Fotografia intraoperatória obtida após ressecção da lesão intra-axial, mostrando a dissecção da vala sílvica. As letras A-D marcam os limites da lesão detetados por neuronavegação. As áreas eloquentes corticais mapeadas antes da ressecção tumoral com eletroestimulação cortical estão marcadas com os números 1, 2, 3 (girus frontal ascendente- área motora) e 4 (pars triangularisárea de linguagem induzindo anomia). 

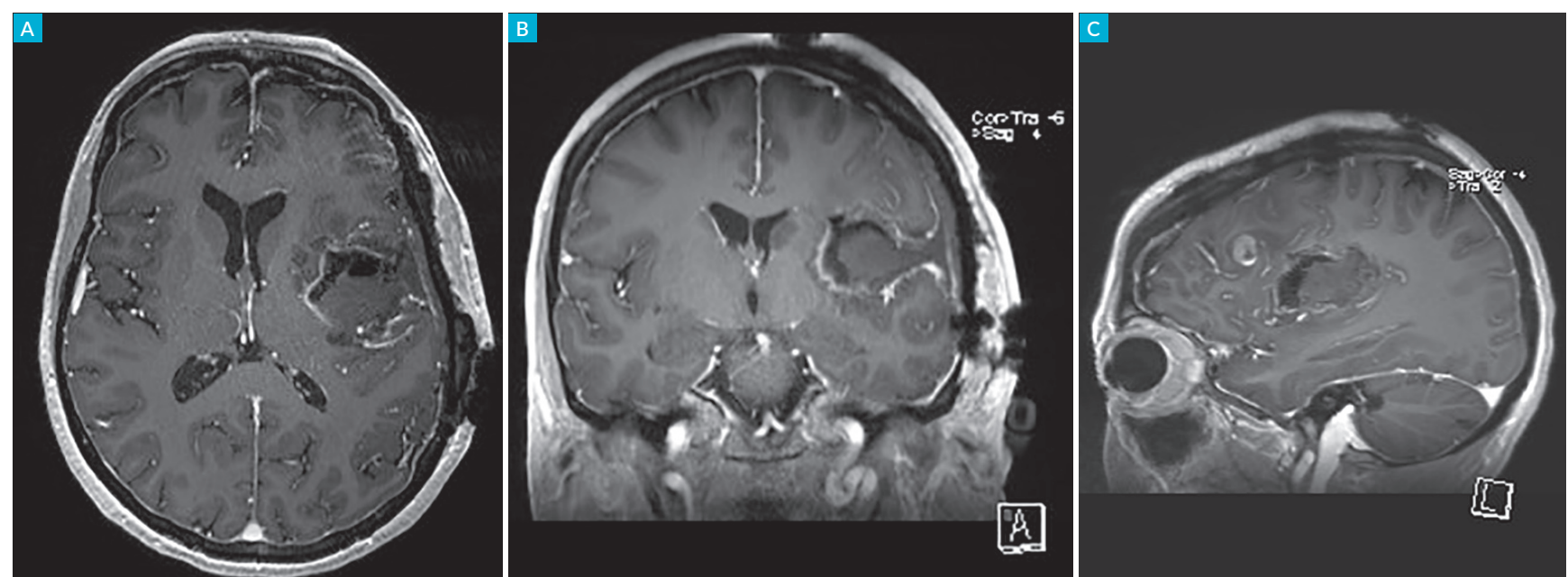

FIGURA 4. RM cranioencefálica pós-operatória (sequência T1 com gadolínio) documentando ressecção total da lesão intra-axial fronto-opercular e insular esquerda em axial (A), coronal (B) e sagital (C).

ção neuropsicológica pré-operatória e de acordo com a localização da lesão, aliada aos conhecimentos de neuroanatomia, nomeadamente a leitura, cálculo, escrita, memória de trabalho, cognição espacial, entre outras.

A cirurgia com o doente acordado constitui uma ferramenta essencial para maximizar a extensão da ressecção cirúrgica, diminuindo a morbilidade do doente.

Nos gliomas de alto grau a ressecção deve ser máxima de acordo com os limites funcionais, aumentando o impacto oncológico, a sobrevida e a qualidade de vida.

CONFLITOS DE INTERESSE: Os autores declaram não ter qualquer conflito de interesse na realização do presente trabalho.

FONTES DE FINANCIAMENTO: Não houve qualquer fonte de financiamento na realização do presente trabalho.

CONFIDENCIALIDADE DOS DADOS: Os autores declaram ter seguido os protocolos da sua instituição acerca da publicação dos dados de doentes.

PROTEÇÃO DE PESSOAS E ANIMAIS: Os autores declaram que os procedimentos seguidos na elaboração

do presente trabalho estão em conformidade com as normas das comissões de investigação clínica e de ética, bem como da declaração de Helsínquia e da Associação Médica Mundial.

CONFLICTS OF INTEREST: The authors declare that they have no conflicts of interest.

FINANCIAL SUPPORT: This work has not received any contribution, grant or scholarship.

CONFIDENTIALITY OF DATA: The authors declare that they have followed the protocols of their work center on the publication of data from patients.

PROTECTION OF HUMAN AND ANIMAL SUBJECTS: The authors declare that the procedures followed were in accordance with the regulations of the relevant clinical research ethics committee and with those of the Code of Ethics of the World Medical Association (Declaration of Helsinki).

\section{REFERÊNCIAS}

1. Castelli J, Feuvret L, Haoming QC, Biau J, Jouglar E, Berger A, et al. Prognostic and therapeutic factors of gliosarcoma from a multi-institutional series. J Neurooncol. 2016;129:85-92.

2. Hervey-Jumper SL, Berger MS. Maximizing safe resection of low- and high-grade glioma. J Neurooncol. 2016;130:269-82.

3. Hervey-Jumper SL, Berger MS. Technical nuances of awake brain tumor surgery and the role of maximum safe resection. J Neurosurg Sci. 2016;59:351-60.

4. Kosac KR, Mahadevan A, Moody JS. Adult gliosarcoma: epidemiology, natural history and factors associated with outcome. Neuro Oncol. 2009;11:183-91.

5. Duffau H, Capelle L, Denvil D, Sichez N, Gatignol P, Taillandier $L$, et al. Usefulness of intraoperative electrical subcortical mapping during surgery for low-grade gliomas located within eloquent brain regions: functional results in a consecutive series of 103 patients. J Neurosurg. 2003;98:764-78.

6. Benzagmout M, Gatignol P, Duffau H. Resection of World Health Organization Grade II gliomas involving broca's area: methodological and functional considerations. Neurosurgery. 2007;61:741-53.

7. Shiban E, Krieg SM, Haller B, Buchmann N, Obermueller T, Boeckh- Behrens T, et al. Intraoperative subcortical motor evoked potential stimulation: how close is the corticospinal tract? J Neurosurg. 2015;123:711-20.

8. Stupp R, Hegi ME, Mason WP, van der Bent MJ, Taphoorn MJ, Janzer RC, et al. Effects of radiotherapy with concomitant and adjuvant temozolamide versus radiotherapy alone on survival in glioblastoma in a randomized phase III study: 5-year analysis of the EORTC-NCIC trial. Lancet Oncol. 2009;10:459-66.

9. Sanai N, Polley MY, McDermott MW, Parsa AT, Berger MS. An extent of resection threshold for newly diagnosed glioblastomas. J Neurosurg. 2011;115:3-8.

10. Lacroix M, Abi-Said D, Fourney DR, Gokaslan ZL, Shi W, DeMonte F, et al. A multivariate analysis of 416 patients with glioblastoma multiforme: prognosis, extent of resection, and survival. J Neurosurg. 2001;95:190-8.

11. De Witt Hamer PC, Robles SG, Zwinderman A, Duffau H, Berger MS. Impact of intraoperative stimulation brain mapping on glioma surgery outcome: a meta-analysis. J Clin Oncol. 2012;30:2559-65. 